\title{
HERA results and their impact for LHC
}

\author{
N. Raičević (on behalf of the H1 and ZEUS Collaborations) ${ }^{a}$ \\ ${ }^{a}$ University of Montenegro, Faculty of Science, Džorža Vašingtona BB, 20000 Podgorica, Montenegro
}

This paper is focused on recent results on inclusive measurements and determination of parton density functions from HERA and their impact for LHC.

\section{Introduction}

In $p p$ collisions at high energy, as planned at the LHC, the final state is produced from interaction of partons from the colliding protons. According to the factorisation theorem [1], a cross section involving hadrons can be decomposed into a short distance part and a long distance part. The short distance or hard scattering part can be calculated perturbatively using the (ultraviolet) renormalisable theory of QCD. The long distance part involves the parton density functions (PDFs), into which the infrared divergences of QCD are absorbed. The PDFs, which are probability densities of partons existing inside the proton, need to be extracted from experimental data. Deep inelastic scattering (DIS) is considered as one of the best tools for the determination of PDFs.

The H1 and ZEUS experiments at the HERA collider study $e^{ \pm} p$ DIS processes in detail and extract PDFs with high precision as will be discussed in this paper.

The kinematics of inclusive deep inelastic electron $^{1}$-proton scattering is described in terms of the variables $Q^{2}$, the four-momentum transfer squared of the exchanged vector boson, Bjorken $x$, the fraction of the momentum of the incoming nucleon carried by the struck quark, and inelasticity $y=Q^{2} / s x$, which is a measure of the energy transfered between the electron and the proton. The centre of mass energy squared, $s$, is given by the electron and the proton beam energies, $s=4 E_{e} E_{p}$. The neutral current (NC) cross section is defined by three proton structure func-

\footnotetext{
${ }^{1}$ The term electron is used to denote both electrons and positrons.
}

tions, $F_{2}, F_{L}$ and $x F_{3}$ as

$\frac{d^{2} \sigma\left(e^{ \pm} p \rightarrow e^{ \pm} X\right)}{d x d Q^{2}}=\frac{2 \pi \alpha^{2}}{x Q^{4}} Y_{+}\left(F_{2} \mp \frac{Y_{-}}{Y_{+}} x F_{3}-\frac{y^{2}}{Y_{+}} F_{L}\right)$,

with $Y_{ \pm}=1 \pm\left(1-y^{2}\right)$. The part in brackets, depending on structure functions, is called NC reduced cross section. In the framework of the perturbative QCD inspired quark parton model, the structure functions can be directly related to the PDFs. At low $Q^{2}, F_{2}$ is the dominant contribution to the cross section which is an electric-charge squared weighted sum of all flavor quark and anti-quark PDFs. In the low $x$ region, $F_{2}$ is dominated by sea-quark PDFs, and the DGLAP [2] evolution of QCD ascribes the $Q^{2}$ dependence of $F_{2}$ ("scaling violation") originating from gluon splitting into $q \bar{q}$ pairs. HERA experiments provide crucial information on the low $x$ sea-quark and gluon PDFs. At large values of $Q^{2}$, $x F_{3}$ becomes significant, and gives information on the valence quark distributions, $u_{v}=u-\bar{u}$ and $d_{v}=d-\bar{d}$. The structure function $F_{L}$ is zero in the quark-parton model, i.e. without QCD, but in leading order QCD, a finite value of $F_{L}$ is expected in the low $x$ region which is directly related to the gluon $\mathrm{PDF}$.

The cross sections of the charged current (CC) DIS interaction, $e^{+}\left(e^{-}\right)+p \rightarrow \bar{\nu}(\nu)+X$, can be written as

$\frac{d^{2} \sigma\left(e^{+} p\right)}{d x d Q^{2}}=\frac{G_{F}^{2}}{2 \pi} \frac{M_{W}{ }^{4}}{\left(Q^{2}+M_{W}^{2}\right)^{2}}\left[\bar{u}+\bar{c}+(1-y)^{2}(d+s)\right]$,
$\frac{d^{2} \sigma\left(e^{-} p\right)}{d x d Q^{2}}=\frac{G_{F}{ }^{2}}{2 \pi} \frac{M_{W}{ }^{4}}{\left(Q^{2}+M_{W}\right)^{2}}\left[u+c+(1-y)^{2}(\bar{d}+\bar{s})\right]$, thus bringing flavor sensitivity of the valence quark PDFs at large $x$.

About $100(20) \mathrm{pb}^{-1}$ of $e^{+} p\left(e^{-} p\right)$ data were collected at HERA until the year 2000 (HERA-I). 
Afterwards, HERA underwent a major upgrade (HERA-II) aiming for higher luminosity, and until March 2007, HERA provided in total about $500 \mathrm{pb}^{-1}$ of $e^{ \pm} p$ collisions per experiment to $\mathrm{H} 1$ and ZEUS. The increased statistics provides an increased sensitivity to the PDFs at large $x$ and large $Q^{2}$, in regions where the precisions of the HERA-I measurements are still statistically limited. In addition, the precision of the structure function measurements by tagging heavy quarks in the final state can be significantly improved by making use of the increased statistics of HERA-II and also the increased capability of heavy quark identification with newly installed tracking devices. During March to June in the year 2007, HERA made a series of dedicated runs with reduced proton beam energies of 460 and $575 \mathrm{GeV}$ as compared to the nominal one $920 \mathrm{GeV}$. These data sets are important for the direct measurement of $F_{L}$, as they allow access to different $y$ for the same $\left(x, Q^{2}\right)$.

Figure 1 shows the kinematic plane in $x$ and $Q^{2}$ covered by the HERA experiments together with the domain probed at LHC. In this figure $y$ represents rapidity, not inelasticity. HERA allows for the first time the study of the proton structure in the perturbative domain at very low $x$, down to $10^{-5}$. This regime is particularly important for LHC physics, since the production of particles with masses of about a few hundred GeV's may involve partons at low $x$. Since the DGLAP equations describe how quark and gluon distribution functions evolve with the scale of the interactions, $Q^{2}$, particle production in the central rapidity region of the LHC can be predicted using the PDFs measured at the same $x$ at HERA.

\section{HERA-I measurements}

A new measurement of the inclusive $\mathrm{NC} e^{+} p$ DIS cross section is obtained by the H1 Collaboration in the region $12 \mathrm{GeV} \leq Q^{2} \leq 150 \mathrm{GeV}^{2}$ and $2 \cdot 10^{-4} \leq x \leq 0.1$ [3]. The results are based on data collected in the year 2000 and combined with previously published data from HERA-I. The accuracy of the combined measurement is typically in the range of $1.3-2 \%$. Figure 2 shows the structure function $F_{2}$ at fixed $Q^{2}$ as a function

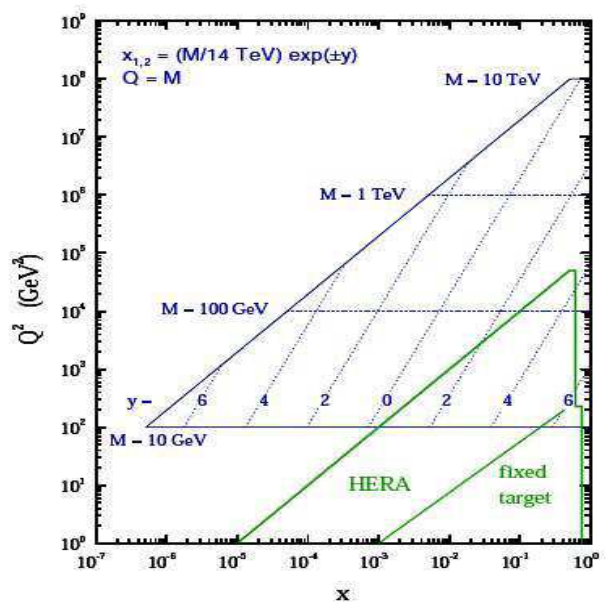

Figure 1. The kinematic plane $x-Q^{2}$ covered by the HERA experiments together with the domain probed at LHC.

of $x$ together with H1 data from lower [4] and higher $Q^{2}$ [5]. The rise of $F_{2}$ when $x \rightarrow 0$ is established at very high accuracy. The data are well described by the newest NLO QCD fit from H1 discussed below.

The NC cross section measurements presented in figure 2 , together with the new measurements at lower $Q^{2}[4]$ and the previously published $\mathrm{H} 1 \mathrm{NC}$ and $\mathrm{CC}$ data provide an accurate $\mathrm{H} 1$ data set for the determination of the PDFs. A new QCD analysis, referred to as H1PDF 2009, is performed [3], which uses a general-mass variable flavour number scheme treatment of the heavy quarks [6]. The general approach to determine the PDFs from experimental DIS cross sections measurements consists of several steps. First, PDFs are parameterised at a low starting scale $Q_{0}{ }^{2}$ by smooth analytical functions with few free parameters. After this, these functions are evolved using the DGLAP equations to higher $Q^{2}$ values and the structure functions and the cross sections are calculated. The calculation is compared to experimental data and a minimisation of the $\chi^{2}$ is performed by adjusting the free parameters. Several constraints can be applied, like momentum sum rules, requiring the known quark flavor numbers of the proton and possibly a few more. The H1PDF 2009 are parameterised at a starting scale 


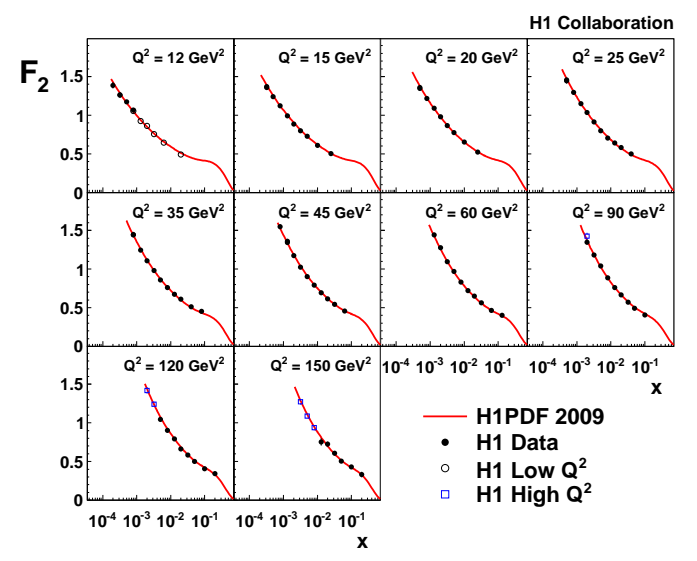

Figure 2. Measurement of the structure function $F_{2}$ at fixed $Q^{2}$ as a function of $x$. The error bars represent the total measurement uncertainties. The curve shows the QCD fit described in the text.

of ${Q_{0}}^{2}=1.9 \mathrm{GeV}^{2}$. Figure 3 shows H1PDF 2009 distributions at $Q^{2}=10 \mathrm{GeV}^{2}$ for the valence distributions for up and down quarks as well as the gluon and sea-quark distributions. The gluon and sea quark $(x g$ and $x S)$ are the dominant parton distributions at low $x$ and are determined with high accuracy due to the high precision of the $F_{2}$ measurement. As can be seen from the figure, the PDF parameterisation uncertainty, resulting from the parameterisation choice, dominates the high $x$ region and the valence distributions, while the low $x$ region is dominated by the model uncertainties which are obtained by varying: the charm mass, the bottom mass, the strange fraction, the minimum $Q^{2}$ used in the fit and the starting scale $Q_{0}{ }^{2}$. The resulting model uncertainty at low $x$ is dominated by the sensitivity of the fit to the $Q_{0}{ }^{2}$ variation.

The cross section measurements are generally affected by statistical and systematic uncertainties. Both $\mathrm{H} 1$ and ZEUS data sets have small statistical errors, thus the systematic uncertainties become increasingly important and their proper treatment essential. The HERA experiments improved this situation by averaging all inclusive $\mathrm{H} 1$ and ZEUS HERA-I published results in a modelindependent way prior to performing a QCD anal-

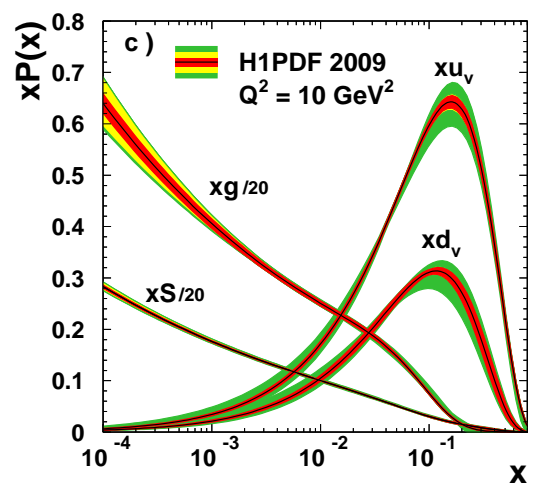

Figure 3. Parton distributions as determined by the H1PDF 2009 QCD fit at $Q^{2}=10 \mathrm{GeV}^{2}$. The gluon and sea-quark densities are scaled down by a factor 0.05. The inner error bands show the experimental uncertainty, the middle error bands include the theoretical model uncertainties of the fit assumptions, and the outer error bands represent the total uncertainty including the parameterisation uncertainty.

ysis on them [7]. Since both experiments measure the same cross section at a given kinematic point, the combination procedure is a $\chi^{2}$ minimization in which the parameters are the true values of the cross section at each $\left(x, Q^{2}\right)$ point and the correlated systematic error parameters [8]. For each measurement point a given correlated uncertainty of one data set of measurements is constrained by the independent measurements from other data sets. Such cross-calibration leads to a precise and coherent data set to be used for the determination of the PDFs.

In the $\chi^{2}$ minimisation procedure the uncertainties can be treated as absolute (additive), so they do not depend on the central value of the measurement, or relative (multiplicative). Normalisation uncertainties, like the luminosity uncertainty, are relative, but for other systematic uncertainties the situation is not so clear. To estimate the sensitivity of the average to this issue, various different treatments of the systematic uncertainties are considered. The extreme assumptions treat all uncertainties as multiplicative, or all as additive, apart from the normalisation uncertainties. An additional systematic 

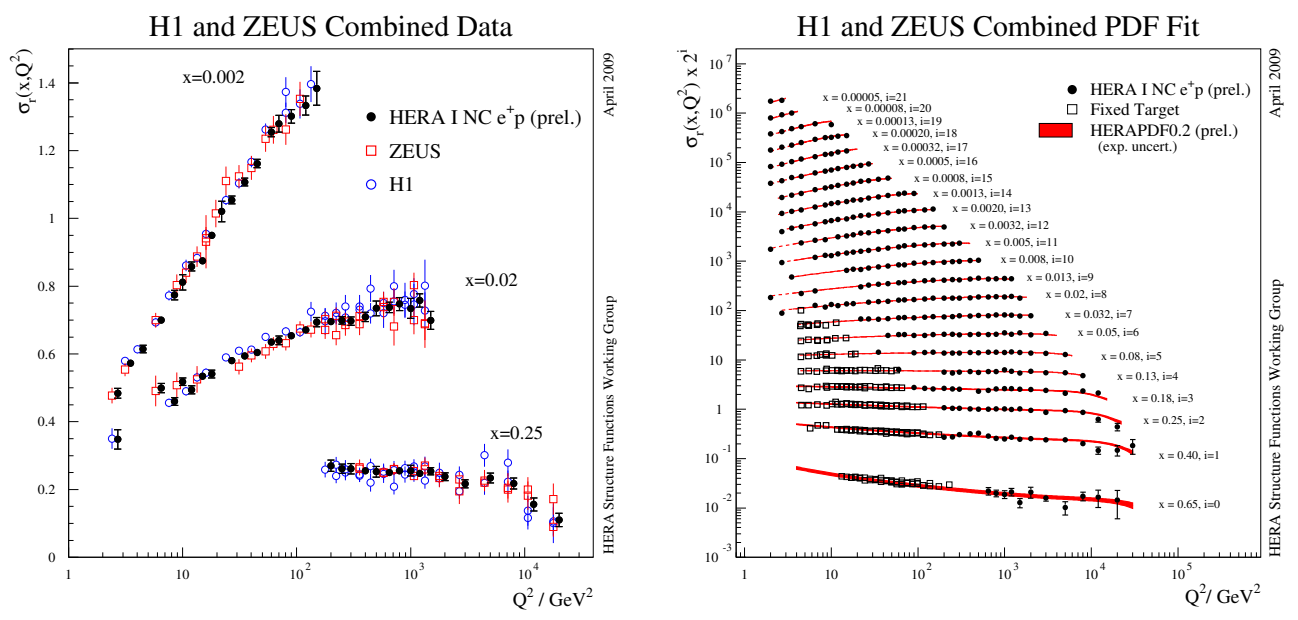

Figure 4. Left: Examples of NC reduced cross section combinations for three bins in $x$ as a function of $Q^{2}$, for H1 and ZEUS and combined experimental points. Right: The combined HERA-I NC reduced cross section compared with the HERAPDF0.2. Both data and fit include the experimental errors.

uncertainty is estimated based on the difference between these two error treatments. The typical size of this uncertainty is less than $0.5 \% .110$ systematic sources from the individual experiments and 3 from the combining procedure are considered. This yields a good quality of the fit with $\chi^{2} /$ dof $=637 / 656$.

Figure 4 (left) illustrates the H1 and ZEUS combined cross sections for three selected $x$ bins as a function of $Q^{2}$. The individual H1 and ZEUS measurements are compared to the $\mathrm{H} 1$ and ZEUS combined measurements. The error bars show the total uncertainty. At low $Q^{2}$ where the measurements are limited by systematic uncertainties, the improvement in the total error is significant. At higher $Q^{2}$ the combined measurements have smaller uncertainties as compared to the individual data due to the increased statistics. In the region $3 \leq Q^{2} \leq 500 \mathrm{GeV}^{2}, 2 \%$ precision for the combined data is obtained, while in the region $20 \leq Q^{2} \leq 100 \mathrm{GeV}^{2}, 1 \%$ precision is reached.

Figure 4 (right) shows the combined HERA-I NC reduced cross section measurements as a function of $Q^{2}$ for various $x$ bins in the extended kinematic range. The measurements are compared to the fixed target measurements and to the fit corresponding to the complete inclusive HERA-I data, the HERAPDF0.2, discussed below. The impressive precision of the combined data is reflected in the precision of the fit. Extrapolated to lower $Q^{2}$, the fit is found to be in very good agreement with the fixed target data.

PDFs are usually determined in global QCD analyses of DIS data, both from HERA and fixed-target experiments, and also jet production data from TEVATRON $[9,10]$. Since high $Q^{2}$ data became available with high statistics, H1 and ZEUS used their own data alone to make QCD fits and extract PDFs $[5,11,3]$. Based on the combined HERA-I dataset of $e^{ \pm} p \mathrm{NC}$ and $\mathrm{CC}$ cross sections presented above, a new NLO QCD fit, HERAPDF0.2, was performed [7]. The HERAPDF0.2 distributions are parameterised at $Q_{0}{ }^{2}=1.9 \mathrm{GeV}^{2}$ with 10 free parameters. The $\chi^{2}$ per degree of freedom of the fit was 576/592.

Figure 5 shows the comparison between the global fits from the MSTW (left) [9] and CTEQ (right) [10] groups and HERAPDF0.2 at $Q^{2}=10 \mathrm{GeV}^{2}$. The global fit distributions are shown at $68 \%$ confidence level. The experimental uncertainties of the HERAPDF0.2 are evaluated at $68 \%$ confidence level and model and parame- 

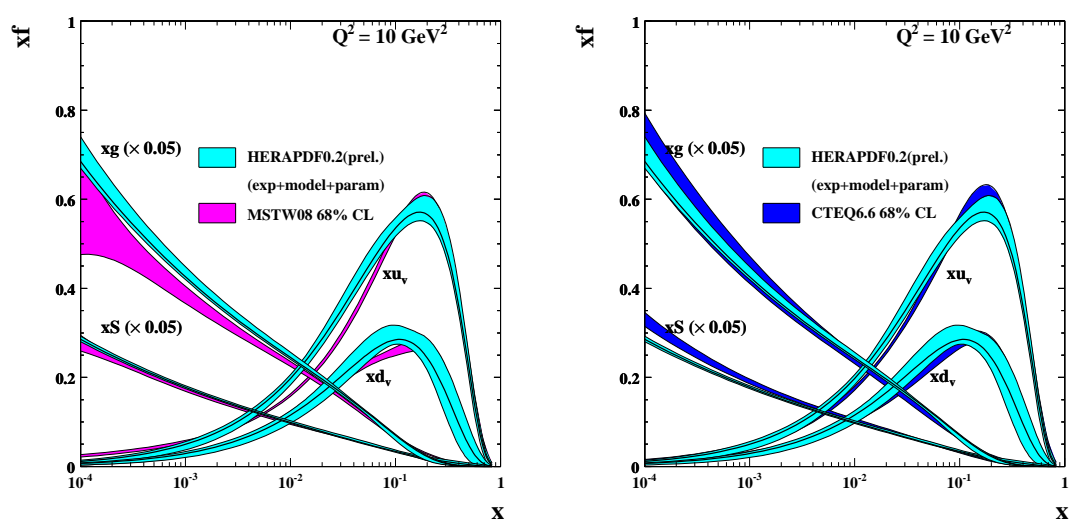

Figure 5. HERAPDF0.2 fit compared with MSTW (left) and CTEQ (right) fits at $Q^{2}=10 \mathrm{GeV}^{2}$.

terisation uncertainties are added. The PDF sets shown are gluon, sea and valence distributions for up and down quarks. The global fits involve data from a much larger variety of experiments and physics processes and thus are forced to use a different error definition than the $\Delta \chi^{2}=1$ criterion applicable to the HERA data alone. The MSTW and CTEQ groups do not evaluate model and parameterisation uncertainties explicitly. The precision of the new data lead to precise PDFs, in particular at low $\mathrm{x}$, where most of the cross-calibration that occurs during the averaging procedure significantly improves the systematic errors of the obtained cross sections.

\section{Further improvements from HERA-II}

The high-statistics HERA-II data will provide a significant improvement of the measurements of CC and NC cross sections at high $Q^{2}$, and thus further improvements are foreseen for valence quark PDFs at large $x$, in particular for the $d$ quark PDF which will be constrained by the $e^{+} p$ CC data. As shown in the ZEUS-JETS fit [11], the medium and high $x$ region $(0.04<x<0.1)$ also profits from measurements of jets which have not been included in the combination of data sets. A measurement of the gluon density at small $x$, which is not obtained from the scaling violations of $F_{2}$, can provide the possibility to investigate the region where the theoretical formalism of the NLO DGLAP equations may need an extension to account for low- $x$ resummation. Such measurements are measurement of $F_{L}$ which is directly related to the gluon, or measurements of $F_{2}{ }^{c \bar{c}}$ and $F_{2}{ }^{b \bar{b}}$ since the heavy quarks are not ordinary constituents in the proton but are produced from the gluon density of the proton.

Measurement of $\mathrm{CC}$ and NC cross sections at high $Q^{2}$. Both collaborations, $\mathrm{H} 1$ and ZEUS, have published or preliminary measurements from $e^{ \pm} p$ interactions on CC and NC cross sections from HERA-II data. The ZEUS Collaboration recently published measurements of the cross sections for CC DIS in $e^{-} p$ collisions with longitudinally polarised electron beams using the whole available statistics from HERA - II data $\left(175 \mathrm{pb}^{-1}\right)$ in the region $280<Q^{2}<30000 \mathrm{GeV}^{2}$ and $0.015<x<0.65$ [12]. The cross section measurement in this kinematic range is shown in figure 6 . The data points were corrected to the unpolarised cross section $\left(P_{e}=0\right)$ using the Standard Model (SM) prediction. The prediction of the SM evaluated using the ZEUS-JETS, CTEQ and MRST PDFs give a good description of the data. The contributions from the PDF combinations $(u+c)$ and $(\bar{d}+\bar{s})$, obtained in the $\overline{M S}$ scheme from the ZEUS-JETS fit, are shown separately and demonstrate the sensitivity to $(u+c)$. ZEUS also has published a new measurement of 

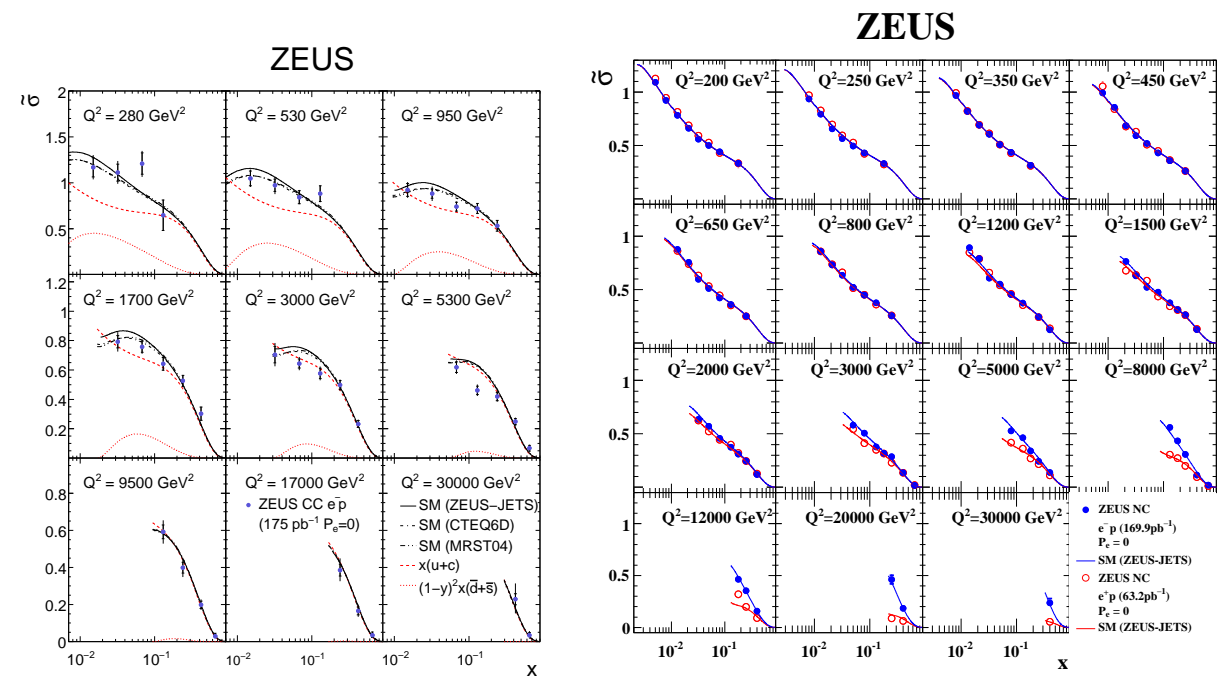

Figure 6. The $e^{-} p$ CC DIS cross section (left) and the $e^{ \pm} p$ unpolarised NC DIS cross section (right) plotted as a function of $x$ at fixed $Q^{2}$.

the NC cross section for DIS in $e^{-} p$ collisions with a longitudinally polarised electron beam [13]. The measurements are based on an integrated luminosity of $169 \mathrm{pb}^{-1}$. The reduced cross sections of unpolarised $e^{-} p$ NC DIS are presented in figure 6 with the residual polarisation of -0.03 corrected using theoretical predictions. The SM predictions are in good agreement with the measurements over the full kinematic range. Also shown are unpolarised $e^{+} p$ NC DIS measurements with an integrated luminosity of $63.2 \mathrm{pb}^{-1}$ collected in 1999 and 2000 [14]. The total systematic uncertainties for most of the phase space used in the reduced cross section measurements are below $2 \%$.

Structure function $x F_{3}$. The difference of the NC cross sections between $e^{+} p$ and $e^{-} p$ collision data determines $x F_{3}$. At HERA, $x F_{3}$ is, to a very good approximation, dominated only by the $\gamma-Z$ interference term, $x F_{3}{ }^{\gamma Z}$, which is determined by the valence quark PDFs and predicted to be only very weakly depending on $Q^{2}$. H1 and ZEUS have combined their data to obtain more precise measurement of $x F_{3}$ [15] based on an integrated luminosity of $489 \mathrm{pb}^{-1}$ which is about one half of the available statistics. With the new $e^{-} p$ $\mathrm{NC}$ reduced cross section, ZEUS updated their $x F_{3}$ measurement [13].

Jet cross section data. The H1 Collaboration has published a new measurement on inclusive jet, 2 -jet and 3 -jet cross sections from $e^{ \pm} p$ scattering at large $Q^{2}, 150<Q^{2}<15000 \mathrm{GeV}^{2}$ using HERA-I and HERA-II data, corresponding to an integrated luminosity of $395 \mathrm{pb}^{-1}$ [16]. The measurements are well described by perturbative QCD calculations at next-to-leading order corrected for hadronisation effects. The strong coupling as determined from these measurements is $\alpha_{s}\left(M_{Z}\right)=0.1168 \pm 0.0007$ (exp. $)_{-0.0030}^{+0.0046}$ (th. $) \pm$ 0.0016 (pdf).

Measurement of the longitudinal proton structure function. The $\mathrm{H} 1$ and ZEUS Collaborations have published measurements of the longitudinal structure function $F_{L}$ at medium $Q^{2}$ determined from $\mathrm{NC}$ cross sections at three different centre-of-mass energies, 318, 251 and $225 \mathrm{GeV}[17,18]$. Published $F_{L}$ from $\mathrm{H} 1$ is measured in the region $2.4 \cdot 10^{-4}<x<0.0036$ and $12<Q^{2}<90 \mathrm{GeV}^{2}$, while ZEUS published their measurement in the region $5 \cdot 10^{-4}<x<0.007$ and $20<Q^{2}<130 \mathrm{GeV}^{2}$. H1 extended their measurements to low [20] and high $Q^{2}$ [19] covering $2.5 \leq Q^{2} \leq 800 \mathrm{GeV}^{2}$ and $x$ between 0.00005 and 0.035 . The values of $F_{L}$ result- 

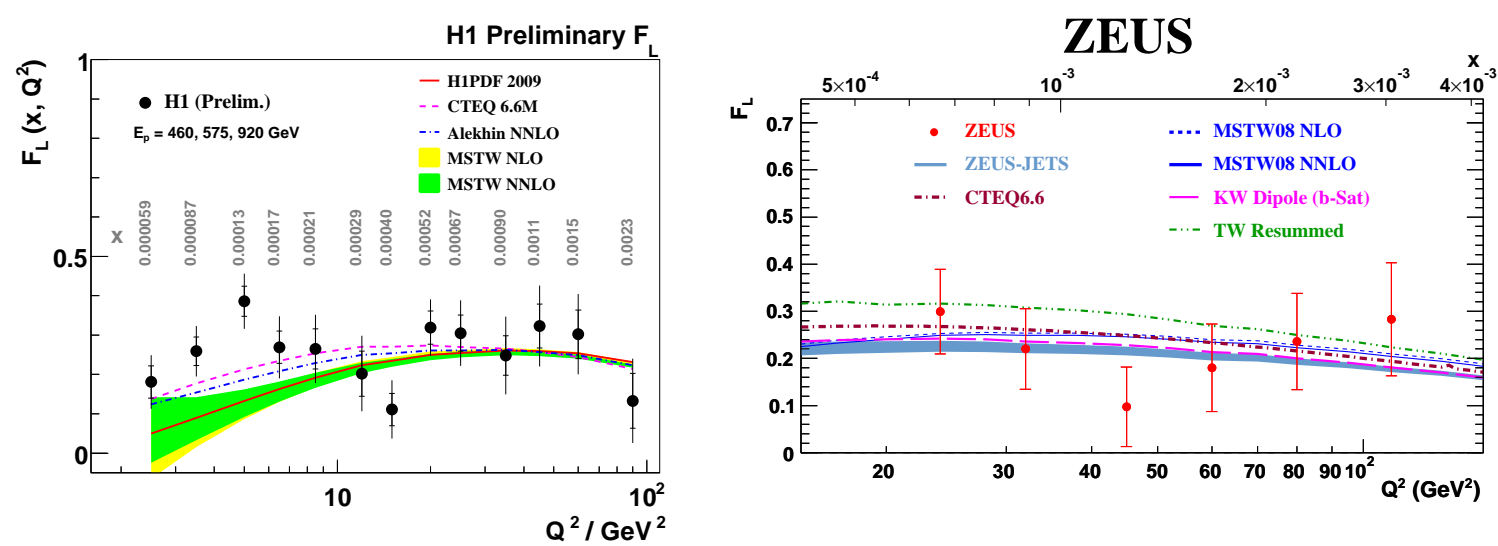

Figure 7. Measurement of the longitudinal structure function $F_{L}$ at H1 (left) and ZEUS (right).

ing from averages over $x$ at fixed $Q^{2}$ are presented in figure 7. The left plot shows results from $\mathrm{H} 1$ and the right one from ZEUS compared with predictions based on the fits to their data alone (H1PDF 2009 and ZEUS-JETS), to CTEQ6.6 [10] NLO and MSTW08 [9] NLO and NNLO fits. H1 compares also with prediction from Alekhin fit at NNLO [21]. All these predictions are based on the DGLAP formalism. Also shown are predictions from the NLL BFKL resummation fit from Thorne and White (TW) [22] and the prediction from the impact-parameterdependent dipole saturation model [23] of Kowalski and Watt based on DGLAP evolution of the gluon density. All of the models are consistent with the data above $10 \mathrm{GeV}^{2}$ (H1) and $24 \mathrm{GeV}^{2}$ (ZEUS). For $Q^{2}<10 \mathrm{GeV}^{2}$ there is a large spread between the models and the data agree better with CTEQ and Alekhin predictions compared to MSTW and H1PDF 2009. H1PDF 2009 and MSTW use the same code to extract $F_{L}$ and underestimate the data. The difference between NLO DGLAP models can be understood as an effect of higher order corrections since the MSTW fit uses terms sub-leading in $\alpha_{s}$ for calculation of $F_{L}$ which turn out to be negative at NLO.

Measurement of the charm and beauty. The heavy quarks are not ordinary constituents in the proton but are produced from the gluon density of the proton. The prediction of the 'standard' QCD processes at the LHC, such as the in- clusive production of $W$ and $Z$ bosons, are sensitive to the theoretical treatment of heavy quarks. The $b$ quark contribution is considered as important in Higgs production at the LHC.

H1 recently published results on inclusive charm and beauty cross sections [24] measured in $e^{-} p$ and $e^{+} p \mathrm{NC}$ interactions in the kinematic region $5 \leq Q^{2} \leq 2000 \mathrm{GeV}^{2}$ and $0.0002 \leq x \leq 0.05$. The analysed data correspond to an integral luminosity of $189 \mathrm{pb}^{-1}$. The charm and beauty events are identified using variables reconstructed by the H1 vertex detector including the impact parameter of tracks to the primary vertex and the position of the secondary vertex. The results on inclusive charm and beauty cross sections with the predictions from $\mathrm{H} 1$ and MSTW at NLO are shown in figure 8 . Both predictions provide a reasonable description of the data.

ZEUS Collaboration also published the new results on charm and beauty production [25] for $Q^{2}>20 \mathrm{GeV}^{2}$.

\section{Summary}

The combined HERA-I data set, of neutral and charged current inclusive cross sections for $e^{+} p$ and $e^{-} p$ scattering, has been used as the input for a NLO QCD fit in the DGLAP formalism. The resulting PDFs are determined with significantly reduced experimental uncertainties compared to the separate analyses of the $\mathrm{H} 1$ and ZEUS experi- 

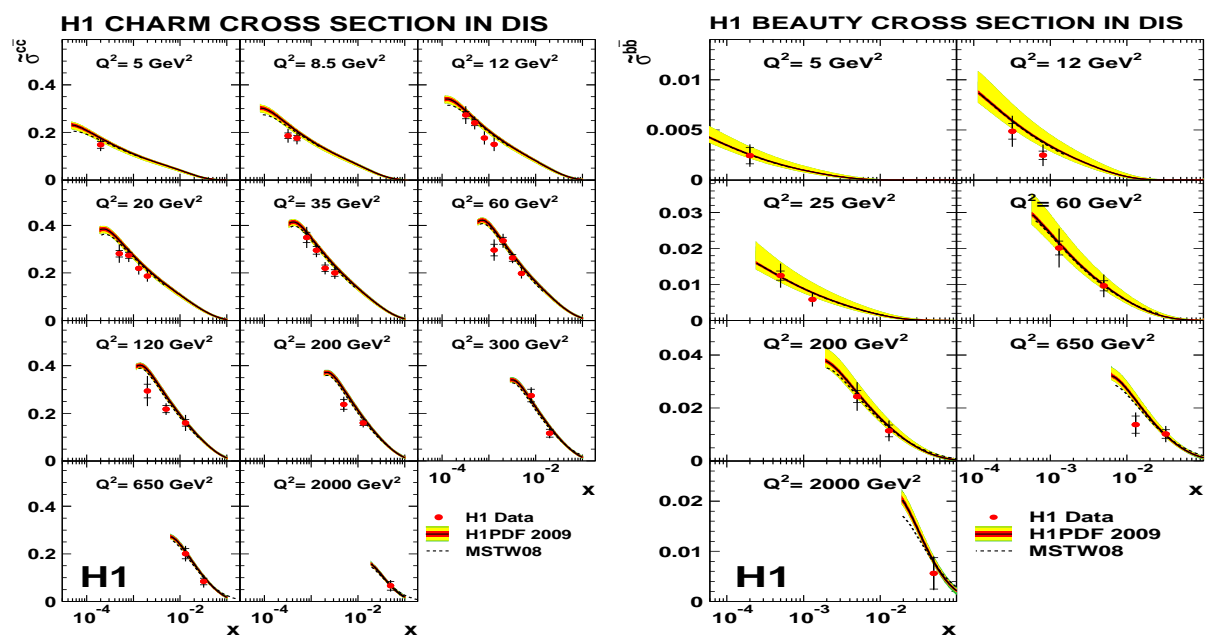

Figure 8. Inclusive charm (left) and beauty (right) cross section as a function of $x$ for different $Q^{2}$ values.

ments. The PDF precision is essential for estimation of the cross sections relevant for LHC. Final publications based on the complete HERA data will come in the next years.

\section{REFERENCES}

1. J.C. Collins, D.E. Soper, G.Sterman, Adv. Ser. Direct. High Energy Phys., volume 5 (1988) 1; hep-ph/0409313.

2. V.N. Gribov and L.N. Lipatov, Sov. J. Nucl. Phys. 15 (1972) 438; V.N. Gribov and L.N. Lipatov, Sov. J. Nucl. Phys. 15 (1972) 675; Y.L. Dokshitzer, Sov. Phys. JETP 46, (1977) 641; G. Altarelli and G. Parisi, Nucl. Phys. B 126 (1977) 298.

3. F.D. Aaron et al., H1 Collaboration, arxiv:0904.3513.

4. F.D. Aaron et al., H1 Collaboration, arxiv:0904.0929.

5. C. Adloff et al., H1 Collaboration, Eur. Phys. J C 30 (2003) 1.

6. R.S. Thorne and R.G Roberts, Phys. Rev. D 57 (1998) 6871.

7. H1 and ZEUS Collaborations, H1prelim-09045 and ZEUS-prel- 09-011.

8. A. Glazov, AIP Conf. Proc. 792 (2005) 237.

9. A.D. Martin, W.J. Stirling, R.S. Thorne and G. Watt, Phys. Lett. B 652, (2007) 292.
10. P.M. Nadolsky et al., Phys. Rev. D 78 (2008) 013004.

11. S. Chekanov et al., ZEUS Collaboration, Eur. Phys. J C 42 (2005) 1.

12. S. Chekanov et al., ZEUS Collaboration, Eur. Phys. J C 61 (2009) 223.

13. S. Chekanov et al., ZEUS Collaboration, Eur. Phys. J C 62 (2009) 625.

14. S. Chekanov et al., ZEUS Collaboration, Phys. Rev. D 70 (2004) 052001.

15. H1 and ZEUS Collaborations, H1prelim-06142, ZEUS-prel-06-022.

16. F.D. Aaron et al., H1 Collaboration, arxiv:0904.3870.

17. F.D. Aaron et al., H1 Collaboration, Phys. Lett. B 665 (2008) 139.

18. S. Chekanov et al., ZEUS Collaboration, arXiv:0904.1092.

19. H1 Collaboration, H1prelim-08-042.

20. H1 Collaboration, H1prelim-09-044.

21. S. Alekhin, JETP Lett. 82 (2005) 628.

22. C.D. White and R.S. Thorne, Phys. Rev. D 75 (2007) 034005.

23. G. Watt and H. Kowalski, Phys. Rev D 78 (2008) 014016 .

24. F.D. Aaron et al., H1 Collaboration, arxiv:0907.2643.

25. S. Chekanov et al., ZEUS Collaboration, arXiv:0904.3487. 